\title{
Future Circular Colliders
}

\author{
Michael Benedikt ${ }^{*}$ \\ CERN, Geneva, Switzerland \\ E-mail: michael.benediktecern.ch
}

Frank Zimmermann

CERN, Geneva, Switzerland

E-mail: frank.zimmermannecern.ch

In response to a request from the 2013 Update of the European Strategy for Particle Physics, the global Future Circular Collider (FCC) study is preparing the foundation for a next-generation large-scale accelerator infrastructure in the heart of Europe.

The FCC study focuses on the design of a 100-TeV hadron collider (FCC-hh), to be accommodated in a new $\sim 100 \mathrm{~km}$ tunnel near Geneva. It also includes the design of a high-luminosity electron-positron collider (FCC-ee), which could be installed in the same tunnel as a potential intermediate step, and a lepton-hadron collider option (FCC-he). The scope of the FCC study comprises accelerators, technology, infrastructure, detectors, physics, concepts for worldwide data services, international governance models, and implementation scenarios.

Among the FCC core technologies figure 16-T dipole magnets, based on $\mathrm{Nb}_{3} \mathrm{Sn}$ superconductor, for the FCC-hh hadron collider, and a highly efficient superconducting radiofrequency system for the FCC-ee lepton collider.

The international FCC study, hosted at CERN, is mandated to deliver a Conceptual Design Report together with a preliminary cost estimate by the time of the next European Strategy Update expected for 2019.

Following the FCC concept—or, more precisely, the preceding "TLEP/VHE-LHC" studies in Europe - IHEP Beijing has initiated the design study for an $e^{+} e^{-}$Higgs factory in China (CEPC), which is to be succeeded by a high-energy hadron collider (SPPC). At present a tunnel circumference of $54 \mathrm{~km}$ and a hadron collider c.m. energy of about $70 \mathrm{TeV}$ are being considered.

This article reports the motivation and the present status of the FCC study, design challenges, R\&D subjects, as well as the emerging global collaboration.

XXVII International Symposium on Lepton Photon Interactions at High Energies

17-22 August 2015

Ljubljana, Slovenia

\footnotetext{
${ }^{*}$ Speaker.

$\dagger$ This work was supported in parts by the European Commission under the Capacities 7th Framework Programme project EuCARD-2, grant agreement 312453, and the HORIZON 2020 project EuroCirCol, grant agreement 654305.
} 


\section{Motivation}

The LHC and its high-luminosity upgrade, the HL-LHC, have an exciting physics programme, which extends through the mid 2030's, i.e. covering the next 20 years.

The original LHC design study was launched in 1983. In other words, it has taken more than 30 years to design, build and commission the LHC and to establish proton-proton collisions at close to design energies. In view of these time scales, it is urgent for the community to start preparing the next accelerator for the post-LHC period, as has clearly been recognized by the 2013 Update of the European Strategy for Particle Physics [1].

A large circular hadron collider seems to be the only approach to reach energy levels far beyond the range of the LHC, during the coming decades, so as to provide access to new particles with masses up to tens of $\mathrm{TeV}$, through direct production, as well as to deliver much increased rates for phenomena in the sub- $\mathrm{TeV}$ mass range, with the corresponding greatly improved precision.

The strong physics case for a future higher-energy hadron collider is reflected in an ICFA statement and in the US P5 recommendations from 2014. The latter confirm that "a very highenergy proton-proton collider is the most powerful tool for direct discovery of new particles and interactions under any scenario of physics results that can be acquired in the P5 time window [1020 years] ...” [2].

European studies in this context started in 2010-2013, for both lepton [3, 4] and hadron colliders [5, 6, 7], under the names LEP3/TLEP and VHE-LHC, respectively. In early 2014 these efforts were combined and expanded as global Future Circular Collider (FCC) study [8].

Meanwhile, on the other side of the world, also in 2014, the Chinese High Energy Physics Association concluded that a "Circular $e^{+} e^{-}$Circular Higgs Factory (CEPC) plus Super $p p$ Collider (SPPC) is the first choice for China's future high energy physics accelerator" [9].

\section{Hadron Collider}

The long-term goal of the FCC study is a 100-TeV hadron collider (FCC-hh), which determines the infrastructure needs of the new facility. The energy reach of a high-energy hadron collider is simply proportional to the dipole magnetic field and to the bending radius: $E \propto B \times \rho$. Assuming a dipole field of $16 \mathrm{~T}$, expected to be achievable with $\mathrm{Nb}_{3} \mathrm{Sn}$ technology, the ring circumference must be about $100 \mathrm{~km}$ in order to reach the target value $100 \mathrm{TeV}$ for the centre-of-mass energy.

The development of the FCC-hh can profit from the design studies for previously considered large hadron colliders, such as the ill-fated Superconducting Super Collider (SSC) in Texas [10], and a Very Large Hadron Collider (VLHC) in Illinois [11].

Figure 1 presents a schematic of the FCC tunnel. Prior to FCC-hh installation, this new tunnel could host a high-luminosity circular $e^{+} e^{-}$collider (FCC-ee). Concurrent operation of hadron and lepton colliders is not foreseen, however. In addition, the FCC study considers aspects of pe collisions, as could be realized, e.g., by colliding the electron beam from an energy recovery linac (ERL) with one of the two FCC-hh hadron beams.

The focus of the Chinese project, proceeding in parallel, is on a circular $e^{+} e^{-}$Higgs factory (CEPC), whose tunnel could later host a hadron collider (SPPC) operating concurrently, and also 


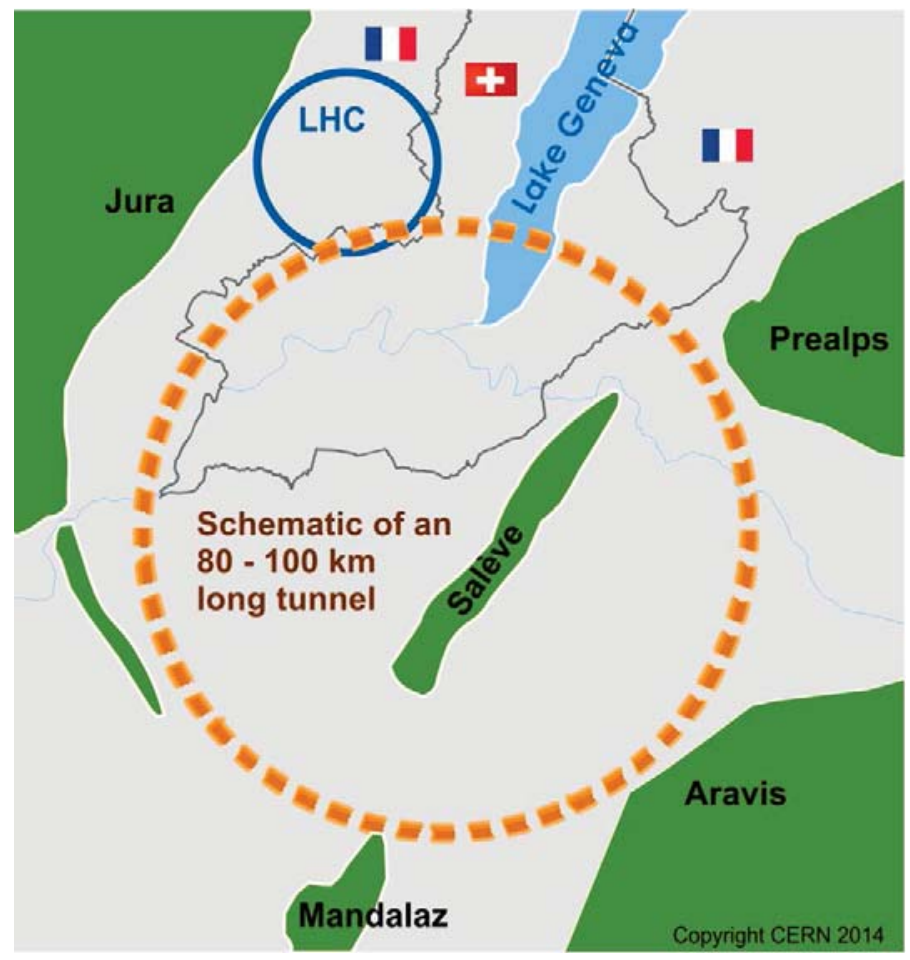

Figure 1: Schematic of a $100 \mathrm{~km}$ tunnel for a Future Circular Collider in the Lake Geneva basin.

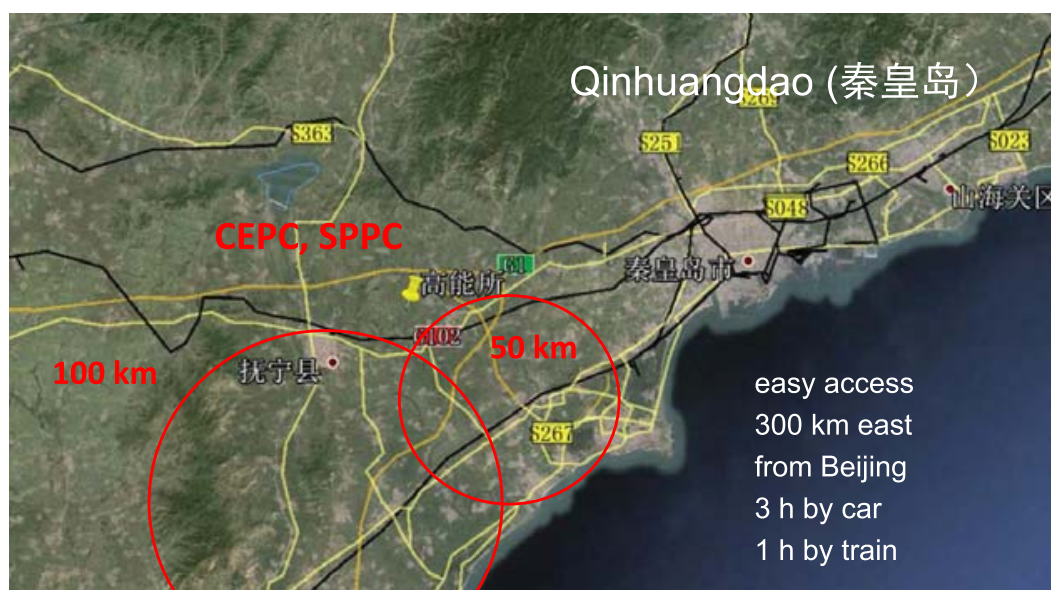

Figure 2: Schematic of 50 or $100 \mathrm{~km}$ tunnel for a Chinese electron-positron Collider (CEPC) and Super proton-proton Collider in Qinhuangdao [12].

allow for ring-ring hadron-lepton collisions [12]. One of the preferred sites of CEPC is Qinhuangdao (see Fig. 2). The CEPC tunnel circumference has preliminarily been chosen to be $54 \mathrm{~km} \mathrm{[13],}$ i.e. substantially smaller than the FCC's. For this reason, the SPPC necessitates a higher dipole field of about $20 \mathrm{~T}$ in order to reach $p p$ collision energies above $70 \mathrm{TeV}$ in the centre of mass. Such a field cannot be realized with $\mathrm{Nb}_{3} \mathrm{Sn}$ coils. Instead the SPPC magnets need to be based on high-temperature superconductor.

Table 1 compares key parameters of FCC-hh and SPPC with those of LHC and HL-LHC. The 
Table 1: Key parameters of LHC, HL-LHC, FCC-hh, and SPPC.

\begin{tabular}{lcccc}
\hline parameter & FCC-hh & SPPC & HL-LHC & LHC $(p p)$ \\
\hline c.m. energy [TeV] & 100 & 71.2 & 14 & 14 \\
ring circumference $[\mathrm{km}]$ & 100 & 54.4 & 26.7 & 26.7 \\
arc dipole field [T] & 16 & 20 & 8.33 & 8.33 \\
number of IPs & $2+2$ & 2 & $2+2$ & $2+2$ \\
initial bunch intensity $\left[10^{11}\right]$ & 1.0 & 2.0 & 2.2 & 1.15 \\
beam current [A] & 0.5 & 1.0 & 1.11 & 0.58 \\
peak luminosity/IP $\left[10^{34} \mathrm{~cm}^{-1} \mathrm{~s}^{-1}\right]$ & $5-29$ & 12 & 5 (levelled) & 1 \\
stored energy per beam [GJ] & 8.4 & 6.6 & 0.7 & $\approx 0.4$ \\
arc synchrotron radiation $[\mathrm{W} / \mathrm{m} /$ aperture] & 28.4 & $\sim 50$ & 0.33 & 0.17 \\
bunch spacing [ns] & 25 or 5 & 25 & 25 & 25 \\
IP beta function $\beta_{x, y}^{*}[\mathrm{~m}]$ & $1.1-0.3$ & 0.75 & 0.15 & 0.55 \\
initial normalized rms emittance $[\mu \mathrm{m}]$ & 2.2 & 4.1 & 2.5 & 3.75 \\
\hline
\end{tabular}

FCC-hh design considers parameter sets for two phases of operation [14, 15]: Phase 1 (baseline) aims at a peak luminosity of $5 \times 10^{34} \mathrm{~cm}^{-2} \mathrm{~s}^{-1}$, and should deliver about $250 \mathrm{fb}^{-1}$ per year on average. In Phase 2 (ultimate) the peak luminosity is increased by almost a factor of six, to $2.9 \times$ $10^{35} \mathrm{~cm}^{-2} \mathrm{~s}^{-1}$, and the integrated luminosity by a factor of four to $1000 \mathrm{fb}^{-1}$ per year. The daily luminosity evolution for these two phases is illustrated in Fig. 3.

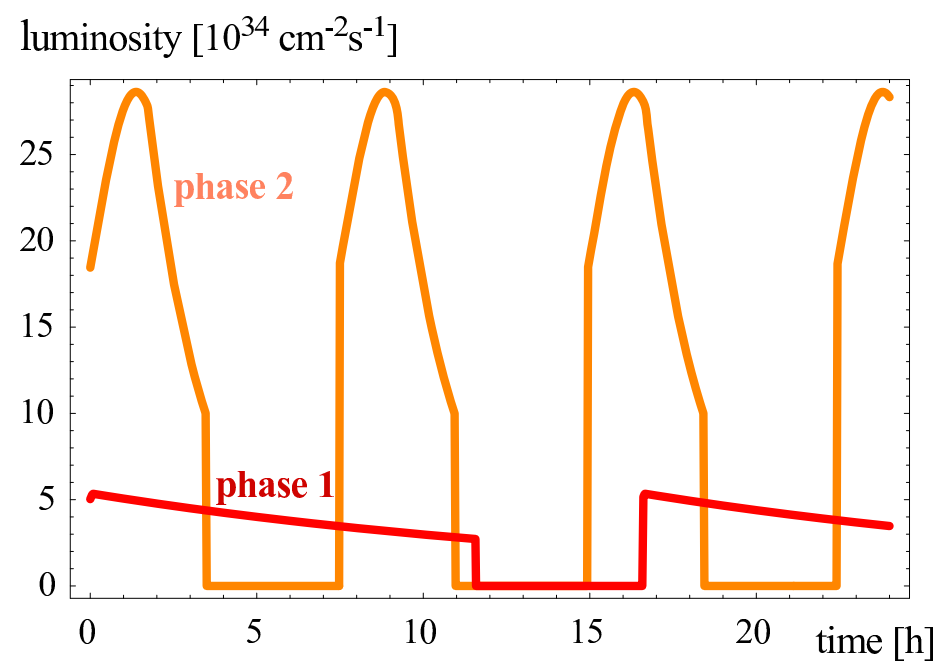

Figure 3: Instantaneous luminosity as a function of time during 24 hours for FCC-hh phases 1 and 2 [15].

The transition from FCC-hh Phase 1 to Phase 2 is realized without any increase in the beam current, primarily by reducing $\beta^{*}$ from 1.1 to $0.3 \mathrm{~m}$, and by accepting a three times larger beambeam tune shift $\left(\Delta Q_{\text {tot }}=0.03\right.$ instead of $0.01[14,15]$; the larger value of 0.03 has been demonstrated at the LHC [16]).

The key physics goals of the FCC-hh are the complete exploration of the Higgs boson and a 
significant extension, via direct and indirect probes, of the search for physics phenomena beyond the Standard Model [17]. Synthesizing the discussions from several theory workshops, an ultimate integrated luminosity goal of $10-20 \mathrm{ab}^{-1}$ for the FCC-hh seems well justified [17]. This goal can be accomplished over 25 years of operation with the two planned FCC-hh phases.

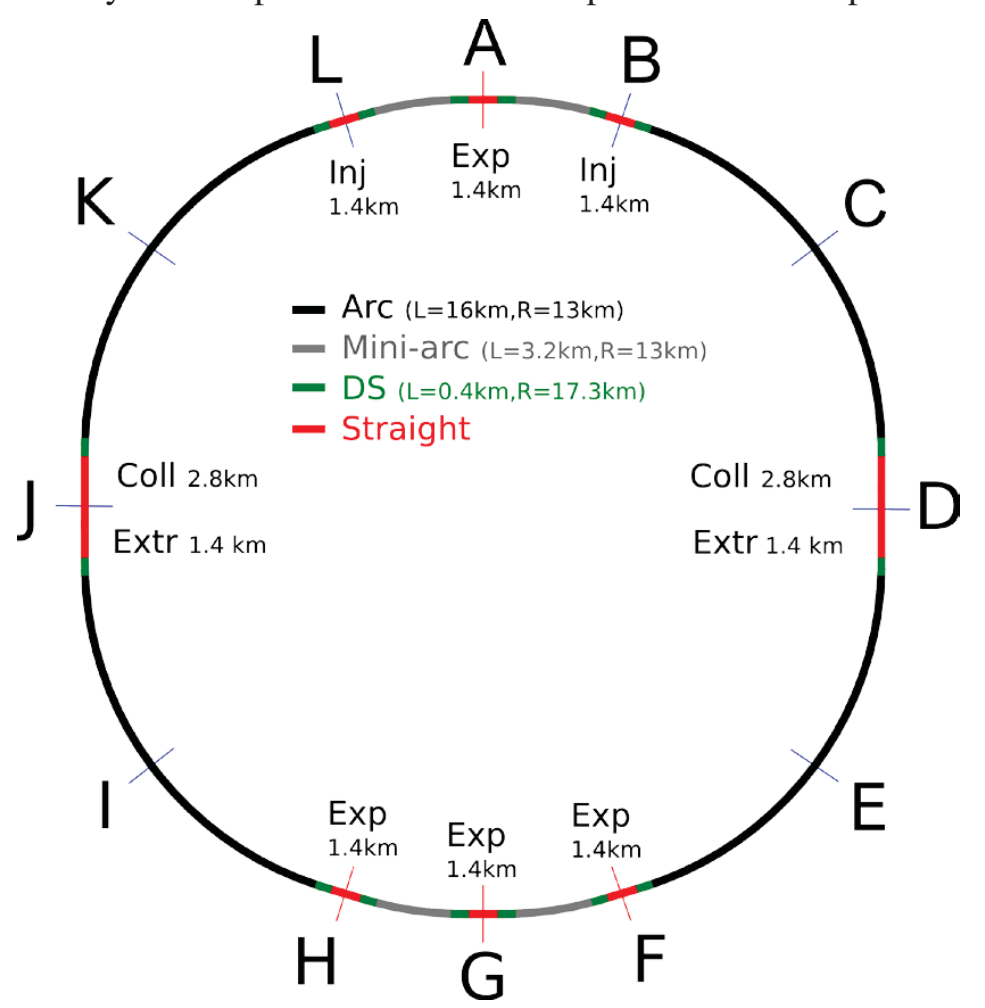

Figure 4: Preliminary layout of FCC-hh [18].

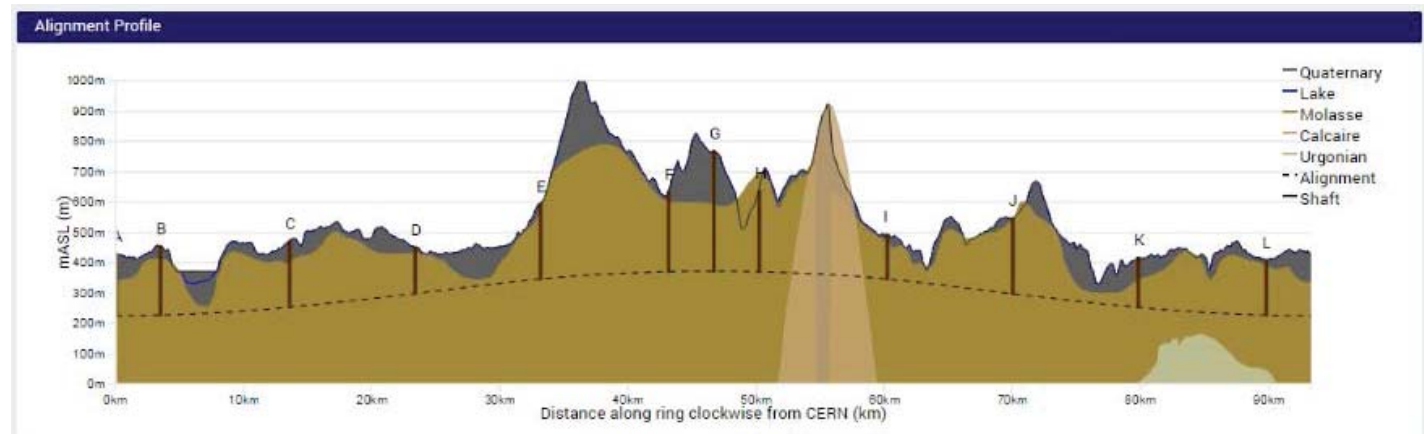

Figure 5: Example placement of a tunnel with $93 \mathrm{~km}$ circumference in the Geneva region, together with geological layers and access shafts; this is a snapshot from a tunnel optimization tool developed in the frame of the FCC study [19].

A preliminary layout for the FCC-hh is shown in Fig. 4. It features two $4.2 \mathrm{~km}$ long straight sections for collimation and beam extraction, as well as six shorter $1.4 \mathrm{~km}$ long straight sections, four of which may accommodate experiments (two high-luminosity and two special-purpose detectors), while the two others serve for injection. 

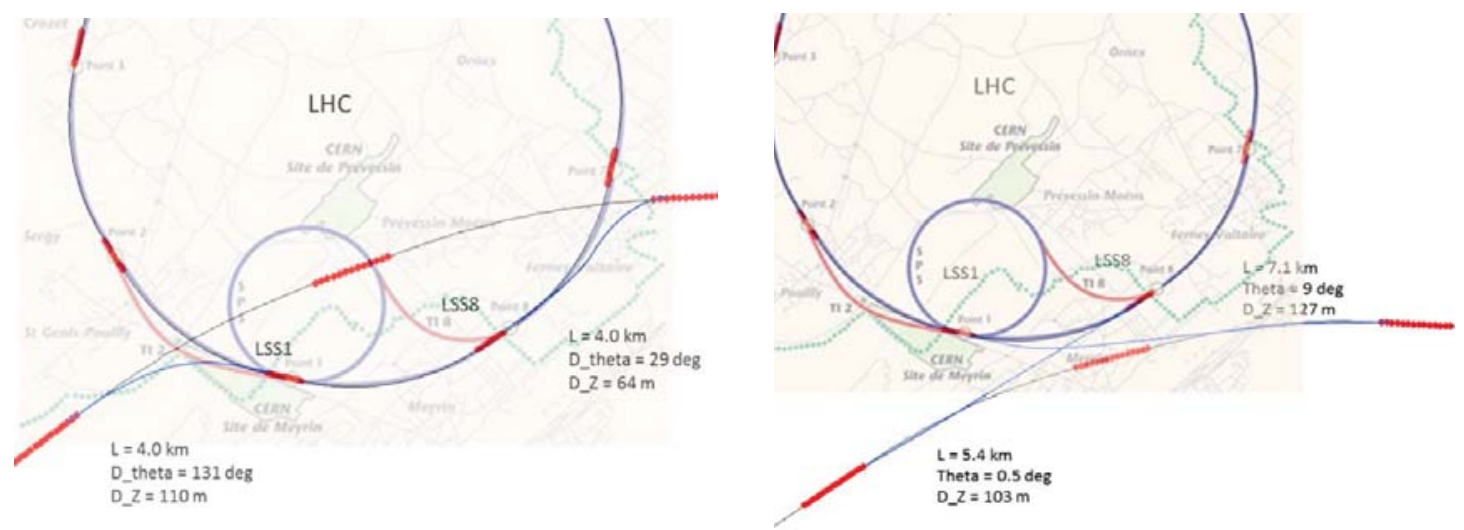

Figure 6: Possible configurations of FCC-hh with LHC used as "High Energy Booster" (HEB) injector [22].

An explorative study of the geology in the Lake-Geneva basin has concluded that a tunnel circumference of 90-100 km would fit the geological situation well (Fig. 5), and that the LHC would be suitable as a potential injector [19]. Using the LHC as "High Energy Booster" for injecting into the FCC-hh also looks feasible from the technical point of view [20, 21]. Two possible configurations of FCC-hh and its LHC injector are illustrated in Fig. 6. For CEPC/SPPC, site investigations are underway in various Chinese provinces, including Qinhuangdao [23].
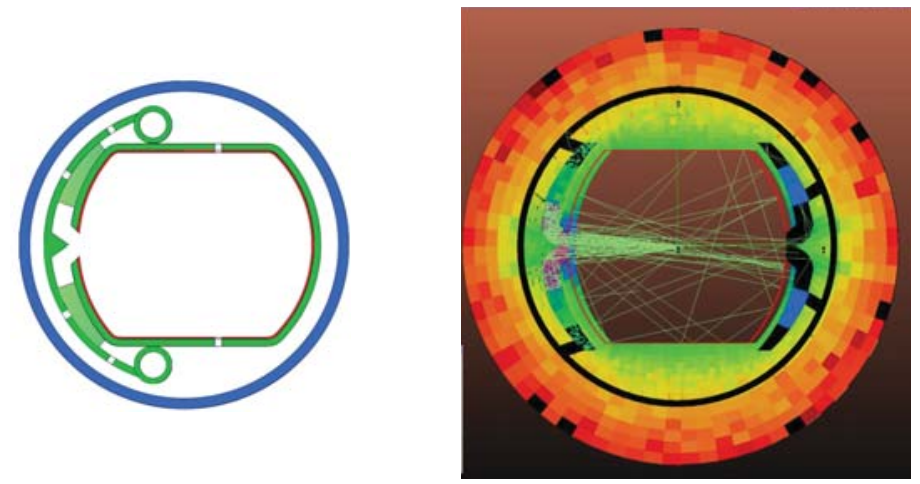

Figure 7: Novel beam-screen design with integrated "folded" antechamber behind a wedge, absorbing most of the synchrotron radiation; asymmetric shape (left) and and alternative symmetric layout together with photon ray tracing (right) $[25,26]$.

The $50 \mathrm{TeV}$ proton beams of the FCC-hh emit substantial amounts of synchrotron radiation (SR), at the level of $30 \mathrm{~W} / \mathrm{m} /$ aperture. This SR may be intercepted by a beam screen (BS) held at a higher temperature, $T_{\mathrm{BS}}$, than the cold bore of the magnets, as already is the case for the LHC, where $T_{\mathrm{BS}} \approx 5-20 \mathrm{~K}$. Contributions to the heat load to be removed by the cryogenic systems include the direct heating of the BS, the cooling of which becomes more efficient at higher $T_{\mathrm{BS}}$, as well as the heat load on the cold bore due to thermal radiation from the BS, which becomes more significant as $T_{\mathrm{BS}}$ increases. For FCC-hh, the optimum value of $T_{\mathrm{BS}}$, which minimizes the total electrical power of the cryogenics plants, lies in the range 50-100 K, depending on the cold bore temperature [24]. In addition, the resistive-wall impedance and vacuum stability may affect the final choice of $T_{\mathrm{BS}}$. Novel BS shapes with an integrated compact antechamber, illustrated in 
Fig. 7 (left), can absorb most of the photons, thereby facilitating the BS cooling and stabilizing the beam vacuum [25]. A more symmetric version of this BS shape (right picture) completely avoids the pumping slots on the inner part, and, thereby, further lowers the beam impedance [26, 27].

In each of the two FCC-hh beams a significant energy of $8 \mathrm{GJ}$ is stored-about 20 times higher than for the LHC, and equivalent to the kinetic energy of an Airbus A380. This has important consequences for machine protection, collimation, beam injection and transfer. The beam transfer for injection is a particularly critical (and unavoidable) process. The number of bunches which can be transferred together will be limited by the associated protection constraints. It increases for a lower injection energy. At top energy a single impacting bunch can destroy a conventional collimator. One of the possible mitigation schemes is the use of indestructible collimators, e.g. hollow-electron lenses [28].

\section{Lepton Collider}

With a circumference of about $27 \mathrm{~km}$, LEP, in operation at CERN from 1989 to 2000, reached a maximum c.m. energy of $209 \mathrm{GeV}$, at a peak SR power around $23 \mathrm{MW}$. The FCC-ee parameters related to energy range and synchrotron radiation represent rather moderate extrapolations from those of LEP2, while the FCC-ee parameters related to luminosity performance resemble those of the more recent B factories (KEKB, and PEP-II). Importantly, SuperKEKB, soon to be commissioned, will demonstrate many of the FCC-ee high-luminosity ingredients.

FCC-ee collisions over a wide range of beam energies, from $35 \mathrm{GeV}$ to $\sim 200 \mathrm{GeV}$, will support extremely high precision tests of the standard model as well as unique searches for rare decays. The FCC-ee physics programme [29] may include: (1) $\alpha_{\mathrm{QED}}$ studies (with energies as low as $35 \mathrm{GeV}$ ) to measure the running coupling constant close to the $Z$ pole; (2) operation on the $Z$ pole (45.5 GeV), where FCC-ee would serve as a "TeraZ" factory for high precision $M_{Z}$ and $\Gamma_{Z}$ measurements and allow searches for extremely rare decays (also enabling the hunt for sterile right-handed neutrinos); (3) running at the $H$ pole $(63 \mathrm{GeV})$ for $H$ production in the $s$ channel, with mono-chromatization, e.g. to map the width of the Higgs; (4) operation at the W pair production threshold $(\sim 80 \mathrm{GeV})$ for high precision $M_{W}$ measurements; (5) operation in $Z H$ production mode (maximum rate of $H$ 's) at $120 \mathrm{GeV}$; (6) operation at and above the $t \bar{t}$ threshold ( $\sim 175 \mathrm{GeV})$; and (7) operation at energies above $175 \mathrm{GeV}$ per beam, should a physics case for the latter be made. Scaling from LEP, at FCCee some beam polarization is expected for beam energies up to about $80 \mathrm{GeV}$ [30], permitting a precise energy calibration.

Some of the key elements of the FCC-ee are: (a) a double ring with separate beam pipes and magnetic systems, magnet-strength tapering (see below), and independent optics control for the counter-circulating electron and positron beams, which intersect each other at two interaction points (IPs) under a total crossing angle of $30 \mathrm{mrad}$; (b) top-up injection based on a booster synchrotron with a cycle period of about $10 \mathrm{~s}$, housed in the same large tunnel, possibly except for bypasses around the particle-physics detectors; and (c) an at least partially local chromatic correction of the final-focus systems.

The range of FCC-ee beam parameters is indicated in Table 2, for simplicity showing numbers of (only) three different operation modes, together with those of CEPC and LEP2. The beam current varies greatly with beam energy, ranging from a few mA, like at LEP2, to $1.5 \mathrm{~A}$, similar 
Table 2: Key parameters for FCC-ee, at three beam energies, and for CEPC, compared with those achieved at LEP2. The FCC-ee parameter refer to a crab-waist scheme with constant, energy-independent arc-cell length [31]).

\begin{tabular}{lccccc}
\hline parameter & & FCC-ee & & CEPC & LEP2 \\
\hline energy / beam [GeV] & 45 & 120 & 175 & 120 & 105 \\
bunches / beam & 60000 & 1400 & 98 & 50 & 4 \\
beam current [mA] & 1450 & 30 & 6.6 & 16.6 & 3 \\
luminosity / IP [10 $\left.\mathrm{cm}^{-2} \mathrm{~s}^{-1}\right]$ & 200 & 9 & 2 & 2 & 0.0012 \\
energy loss / turn [GeV] & 0.03 & 1.67 & 7.55 & 3.11 & 3.34 \\
synchrotron power [MW] & 100 & 100 & 100 & 104 & 22 \\
RF voltage [GV] & 0.2 & 3.6 & 11 & 6.9 & 3.5 \\
\hline
\end{tabular}

to the $\mathrm{B}$ factories. As a design choice, the total synchrotron radiation power has been limited to $100 \mathrm{MW}$, about 4 times the synchrotron-radiation power of LEP2, on all FCC-ee operation points. For a roughly four times larger machine this results in comparable radiation power per unit length. The estimated luminosity numbers scale linearly with the synchrotron-radiation power. Figure 8 displays the expected luminosity per IP as a function of c.m. energy, assuming crab-waist collisions [31] at two interaction points. The anticipated luminosity performance has been confirmed by beam-beam simulations [36, 37]. Recent optics designs for the full ring, with $\beta_{y}^{*}=2 \mathrm{~mm}$, provide an adequate off-momentum dynamic aperture.

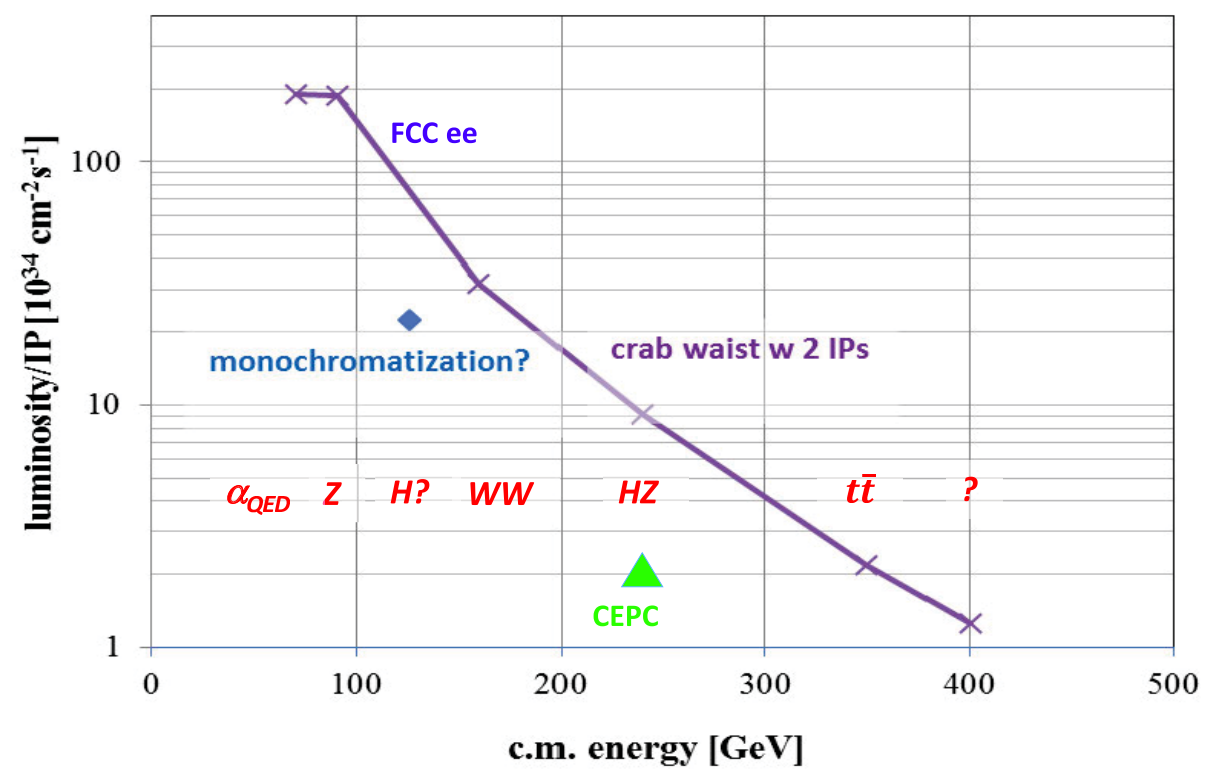

Figure 8: Projected FCC-ee and CEPC luminosity per interaction point (IP) as a function of centre-of-mass energy. cepc

Two optics versions for the final-focus system are illustrated in Fig. 9. Both systems feature a local chromatic correction in the vertical plane, and provide for a crab-waist collision scheme. 

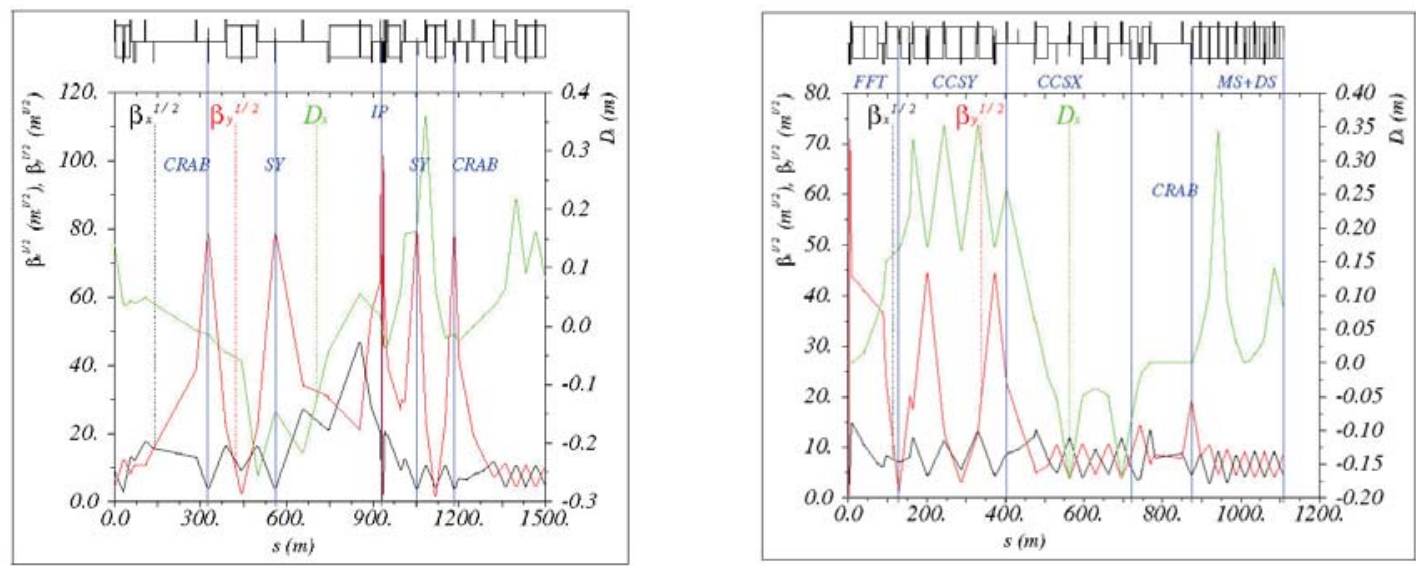

Figure 9: Two alternative optics for the FCC-ee final-focus system, plotted with $\beta_{x}^{*}=1 \mathrm{~m}$ and $\beta_{y}^{*}=2 \mathrm{~mm}$ : asymmetric layout with two sextupoles per side of IP and low photon energies on the incoming side (K. Oide) [34] and symmetric with five sextupoles per side and smaller peak separation, but more energetic photons (A. Bogomyagkov) [35].

The system shown on the left uses a total of two sextupoles only, the second of which cancels the geometric aberrations of the first, and, at reduced strength, also creates a "virtual" crab waist. In this version the horizontal chromaticity of the final focus is corrected by adjusting the strengths of numerous sextupole pairs in the arcs. The system is asymmetric, in that the incoming side includes only weak bending magnets with a critical photon energy below $100 \mathrm{keV}$ on the last $500 \mathrm{~m}$ before the collision point, while the outgoing side has stronger dipoles. By contrast, the system on the right is perfectly symmetric, with critical photon energies up to $400 \mathrm{keV}$. It comprises five sextupoles, two each for vertical and horizontal chromatic correction, and an additional sextupole generating the crab waist. The larger photon energies on the incoming side are a concern as, through neutron production, they may affect the particle-physics detector. On the other hand, an advantage of the symmetric system is that the maximum distance between the two beam lines is reduced, by roughly a factor of two compared with the asymmetric design.

To support the high target luminosity with its associated short beam lifetime, top-up injection is an essential ingredient of the FCC-ee. Promising options to realize this top up include conventional off-momentum injection and multipole-kicker injection [32, 33].

Figure 10 presents one possible FCC-ee collider layout compatible with the geometry of the hadron collider FCC-hh, which features two collision points at opposite positions of the ring. The incoming beam line is bent less than the outgoing beam line in order to minimize the synchrotron radiation emitted in the direction of the experimental detector [38]. This leads to a rather large separation of the inner and outer beam lines on each side of each interaction point (IP). The inner tunnel, corresponding to the hadron collider, as sketched in the figure, might accommodate the detector-bypass for the FCC-ee booster ring. The outer and inner beam lines of the FCC-ee collider cross in the long straight sections half way between the two experiments. In these two long straight sections, the rf systems are located. For $t \bar{t}$ operation, which requires the maximum rf voltage, with only a few tens of bunches, the rf systems are common to the two beams, while at lower beam energies, with high currents and 1000's of bunches, they are separate. For FCC-ee 


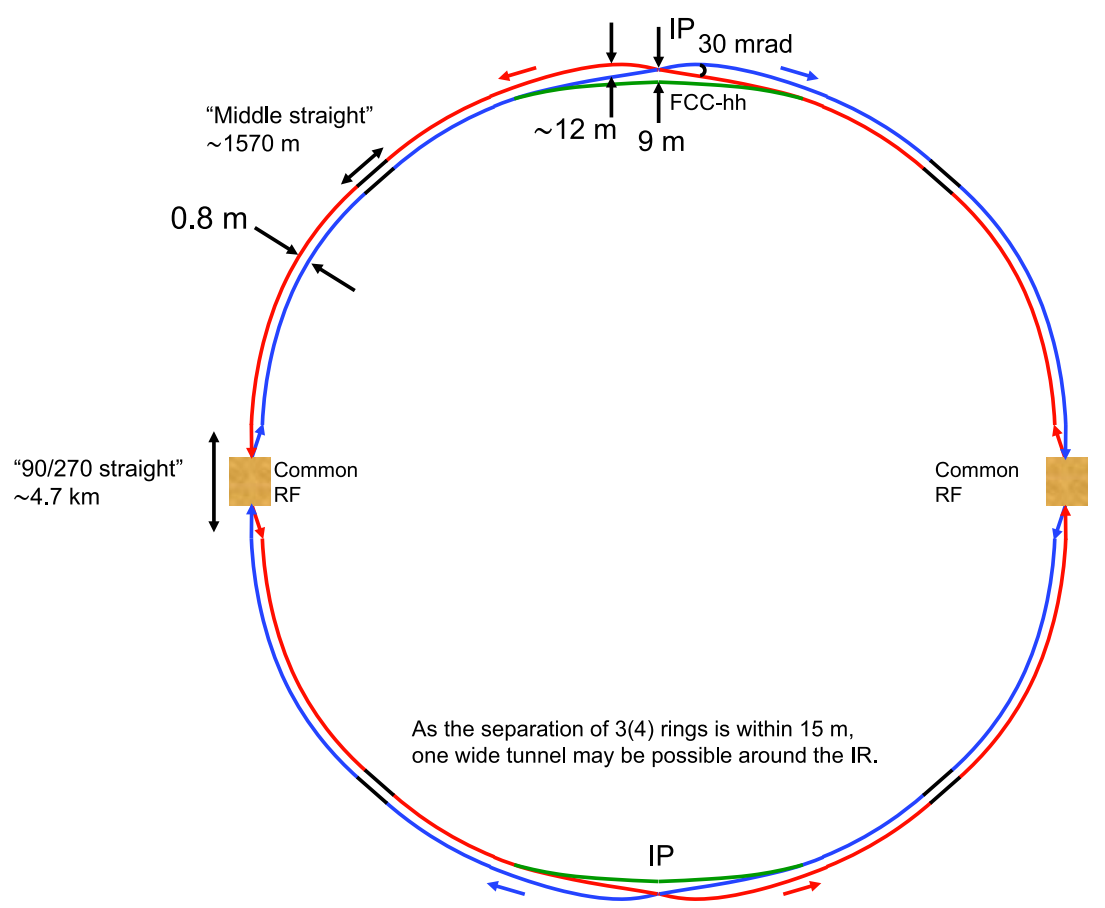

Figure 10: Layout for one possible FCC-ee optics design compatible with the FCC-hh geometry [34].

in $t \bar{t}$ operation the energy variation around the ring is significant. The resulting energy sawtooth induced by synchrotron radiation will be compensated by adjusting the strength of the magnets to the local beam energy. Such a tapering of the dipole and quadrupole magnets can be accomplished through separate powering per half arc, or, more locally, by means of parallel shunts in the magnetcircuits. In the common rf section this type of tapering is not possible, and, here, a simultaneous (symmetric) optics matching for the two beams is performed, similar to the procedure applied for energy recovery linacs [34].

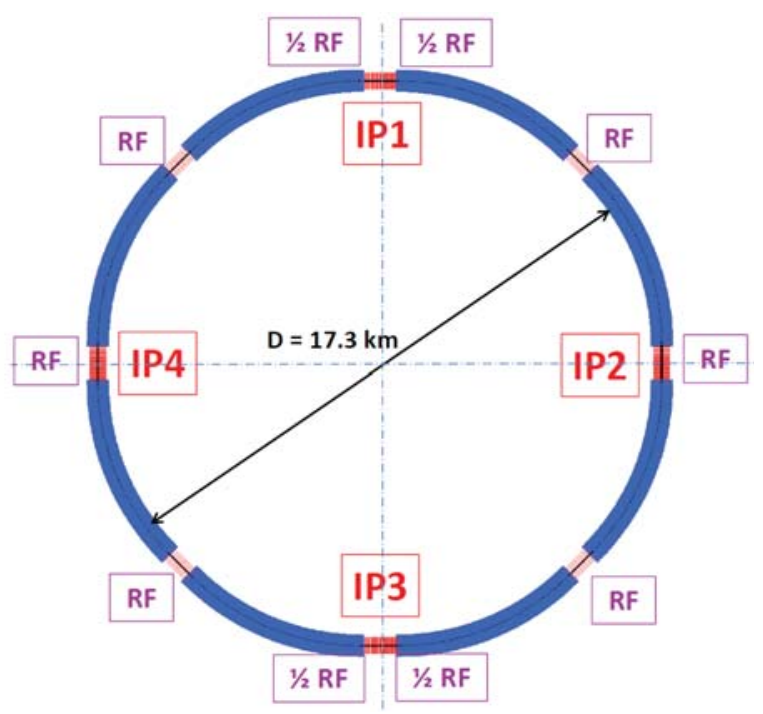

Figure 11: CEPC layout [12]. 
The layout of CEPC is illustrated in Fig. 11. Electrons and positrons are sharing the same beam pipe, preventing the tapering of the magnets, and, as a result, the rf system is distributed over all 8 straight sections.

\section{Lepton-Hadron Option}

An FCC-he lepton-hadron collider may consist of a high-current low-emittance electron beam, provided by an energy-recovery linac (ERL), which collides with the hadron beam circulating in one of the two FCC-hh rings, similar to the proposed LHeC [39], where ERL electrons scatter off an LHC hadron beam. Indeed, the same ERL could first be employed for the $\mathrm{LHeC}$ and later for the FCC-he. In addition to exploring new regimes of QCD, high parton densities, and deep inelastic scattering, the FCC-he physics programme [39] includes measurements of the Higgs self-coupling, as well as precision measurements of $H-b b$ coupling in $W W-H$ production.

Differently from the FCC, the CEPC and SPPC rings are supposed to be installed together in the same tunnel, and could, therefore, be operated concurrently. This coexistence allows for high-luminosity electron-proton ring-ring collisions [12].

\section{Key Technologies}

The key technology R\&D for FCC-hh comprises the superconductor (SC) development and the high-field magnet design. The conductor development aims at a 50\% higher critical current density than achieved for the HL-LHC (see Fig. 12). As for the magnets themselves, a five-year development programme has been launched at CERN [41, 42] (Fig. 13).

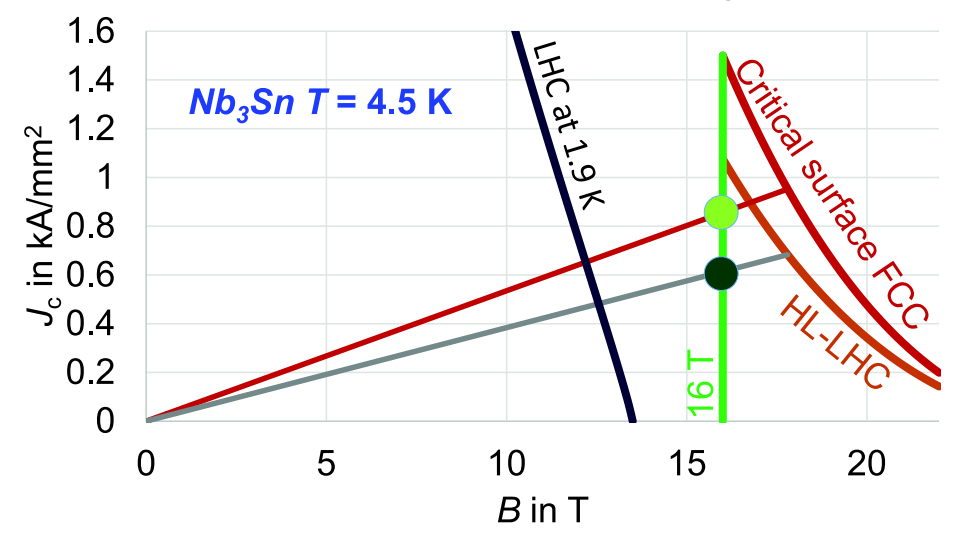

Figure 12: Critical current density versus magnetic field for the HL-LHC $\mathrm{Nb}_{3} \mathrm{Sn}$ conductor at $4.5 \mathrm{~K}$ (lower red curve), and the FCC-hh target performance (upper red curve) together with the associated $16 \mathrm{~T}$ operating points at $10 \%$ margin (the black and green circle, respectively) [41, 42]. For completeness, the criticial current of the present LHC Nb-Ti superconductor at $1.9 \mathrm{~K}$ is also displayed (the black curve).

Parallel efforts are underway to establish complementary programmes in the United States, Japan, and other countries, in order to explore different SC-wire production lines and different coil layouts (e.g. $\cos \theta$ coil [43], block coil [44], and canted $\cos \theta$ coil [45]). Already in September 2015 a small $\mathrm{Nb}_{3} \mathrm{Sn}$ racetrack model at CERN exceeded a field of $16 \mathrm{~T}$ at the coil [46]. While the FCChh design relies on $\mathrm{Nb}_{3} \mathrm{Sn}$ technology, the SPPC's $20 \mathrm{~T}$ accelerator dipole magnets must include a 


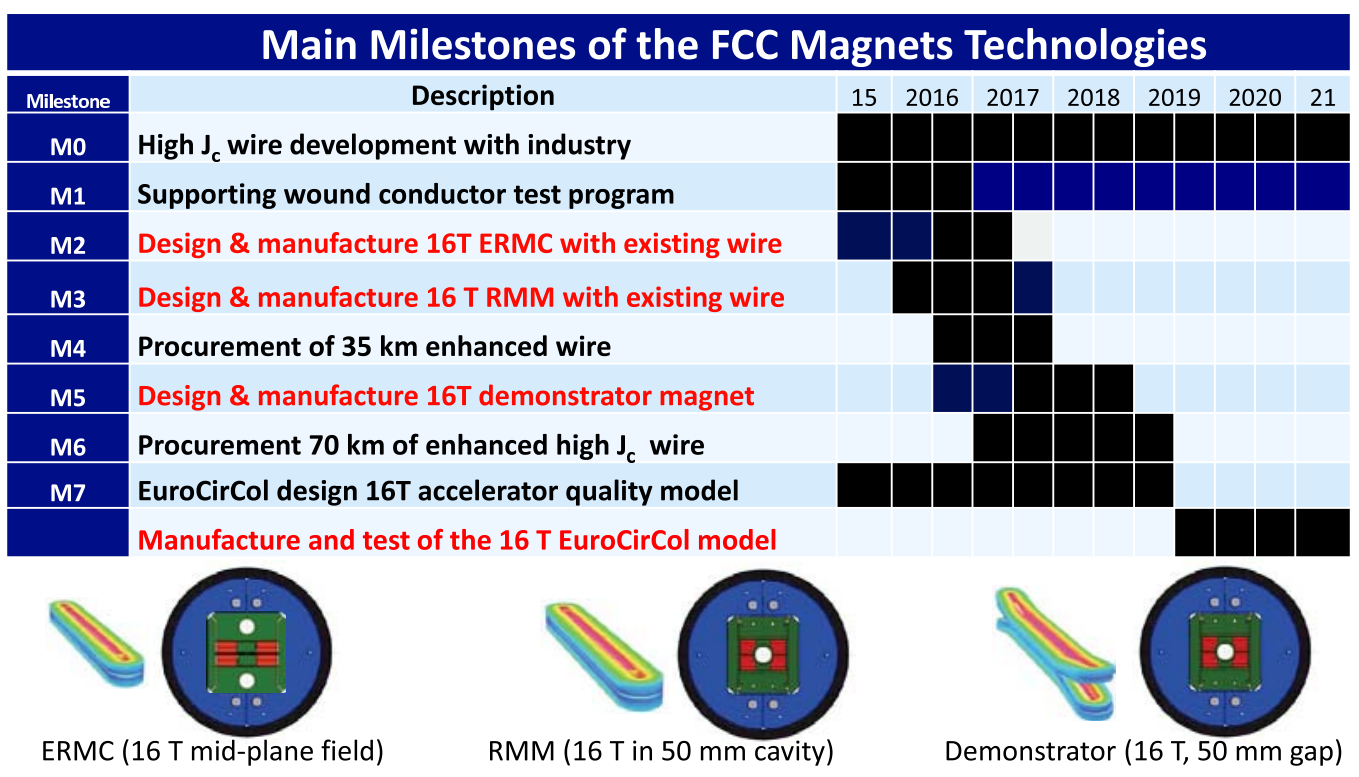

Figure 13: Time line of FCC magnet technology program [41, 42].

significant portion of high-temperature superconductor, for which both Bi-2212 or YBCO are being considered [12]. The coil shape also differs in that the SPPC magnets are made from racetrack coils.

An important technological component of the lepton colliders FCC-ee and CEPC is their superconducting radiofrequency (RF) system. The CEPC will operate at moderate beam current and moderate RF voltage, for which a state-of-the-art system appears suitable [12]. By contrast, the RF requirements for FCC-ee [40] are determined by the following two regimes: (1) high gradients for $\mathrm{H}$ and $t \bar{t}$ when operating with a few tens of bunches, and (2) high beam loading with currents of about $1.5 \mathrm{~A}$ at the $Z$ pole. The FCC R\&D aims at a conversion efficiency from wall-plug power to beam power (roughly equal to the SR power) of $70 \%$ or higher, e.g. by means of innovative klystron design [47].

\section{Collaboration and Time Line}

Since February 2014, a total of 63 institutes from 24 countries and four continents have joined the FCC collaboration. The FCC study aims at delivering a conceptual design report and cost estimates for all collider options by the end of 2018. In early 2015 the FCC study was recognized by the European Commission through the funding of the FCC technical design study (EuroCirCol) via the programme of HORIZON2020. The multipurpose laboratory KEK in Japan and sixteen beneficiaries from the European Research Area committed to perform the core of the FCC-hh collider ring design and the $16 \mathrm{~T}$ magnet $\mathrm{R} \& \mathrm{D}$. The four key themes addressed are the arc design (led by CEA Saclay), the interaction-region design (John Adams Institute), the cryo-beam-vacuum system (ALBA-CELLS), and the high-field magnet design (CERN). Four major U.S. laboratories (BNL, NHMFL, FNAL, LNBNL) are associated with EuroCirCol. 


\section{Conclusions}

Preparation for the post-LHC period is now urgently needed. The FCC study heralds a bright long-term future of accelerator-based high energy physics.

A circular hadron collider appears to be the only path available in this century towards direct discoveries at energies of 10's of TeV. FCC-hh profits from, and extends, the new $\mathrm{Nb}_{3} \mathrm{Sn}$ technology of the HL-LHC. In addition, it promotes numerous other technological innovations. SPPC requires a novel accelerator-magnet technology based on high-temperature superconductor.

FCC-ee is an attractive intermediate step towards FCC-hh, just as CEPC is towards SPPC. The lepton and hadron collider projects are also highly synergetic: FCC-ee (or CEPC) may share the infrastructure, cryogenics systems, and RF developments with FCC-hh (respectively, SPPC). FCC-ee (CEPC) would also allow for an exciting physics programme during the fabrication of the FCC-hh (SPPC) magnets, and it may provide indications for new physics later to be explored at the FCC-hh (SPPC). FCC-he supports fully complementary physics studies, and could be realized as an extension of the LHeC, while CEPC/SPPC electron-proton collisions can be realized by intersecting these two rings, which will be operating concurrently.

There is a great worldwide interest in the FCC and CEPC/SPPC studies. A global collaboration is being formed and acquiring momentum towards the FCC goals [48]. An internationalization of CEPC/SPPC is also being pursued.

\section{Acknowledgements}

This report summarizes work performed by the international FCC collaboration and by the CEPC/SPPC team. We thank the FCC study coordination group and all collaboration members for their excellent contributions to the FCC design study.

\section{References}

[1] European Strategy Session of Council, 30 May 2013, CERN-Council-S/106 (2013).

[2] S. Ritz et al., Building for Discovery, US P5 Report, May 2014.

[3] A. Blondel, F. Zimmermann, A High Luminosity $e^{+} e^{-}$Collider in the LHC Tunnel to Study the Higgs Boson, 12 December 2012, arXiv:1112.2518 [hep-ex] .

[4] EuCARD LEP3 workshop, 18 June 2012, http: / / indico. cern. ch/event/193791; 2nd EuCARD LEP3 workshop, 23 October 2012, http : / / indico. cern. ch/ event/211018; 3rd EuCARD TLEP3 workshop, 10 January 2013, http: / / indico. cern. ch/event/222458; 4th EuCARD TLEP workshop, 4-5 April 2013, http: / / indico. cern. ch/event/240814.

[5] E. Todesco and F. Zimmermann (eds.), Proc. EuCARD-AccNet-EuroLumi Workshop: The High-Energy Large Hadron Collider - HE-LHC10, Malta, 14-16 October 2010, CERN-2011-003, arXiv:1111.7188 [physics.acc-ph].

[6] J.A. Osborne, C.S. Waaijer, Pre-Feasability Assessment for an $80 \mathrm{~km}$ Tunnel Project at CERN, Contribution to the Update of the European Strategy for Particle Physics, No. 165, 27 July 2012 , http://indico.cern.ch/event/175067/call-for-abstracts/165/file/1.pdf. 
[7] Joint Snowmass-EuCARD/AccNet-HiLumi meeting 'Frontier Capabilities for Hadron Colliders 2013' a.k.a. EuCARD VHE-LHC Day, CERN, 21-22 February 2013,

http://indico.cern.ch/event/223094.

[8] Future Circular Collider Study Kickoff Meeting, University of Geneva, 12-15 February 2014, http://indico.cern.ch/e/fcc-kickoff.

[9] J. Gao, The Strategy of Accelerator Based High Energy Physics of China, Rencontres du Vietnam, 10-17 August 2014

[10] J.D. Jackson (ed.), Superconducting Super Collider Conceptual Design Report, SSC-SR-2020 (1986).

[11] The VLHC Design Study Group, Design Study for a Staged Very Large Hadron Collider, Fermilab-TM-2149, SLAC-R-591 (2001).

[12] A. Apyan et al., CEPC-SPPC Preliminary Conceptual Design Report, IHEP-CEPC-DR-2015-01, IHEP-AC-2015-01 (2015).

[13] Y. Zhang, H. Geng, Y. Wang and D. Wang, Status and Challenges of CEPC Accelerator Design, ICFA Beam Dynamics Newsletter No. 67, August 2015, http: / / www-bd. fnal.gov/ icfabd.

[14] M. Benedikt et al., FCC-hh Hadron Collider - Parameter Scenarios and Staging Options, Proc. IPAC'15 Richmond (2015) 2173.

[15] M. Benedikt, D. Schulte, F. Zimmermann, Optimizing Integrated Luminosity of Future Hadron Colliders, Phys. Rew. ST - Accel. Beams 18, 101002 (2015).

[16] W. Herr et al. Observations of beam-beam effects at high intensities in the LHC, Proc. IPAC'11 San Sebastian (2011) 1936.

[17] I. Hinchliffe, A. Kotwal, M.L. Mangano, C. Quigg, L.-T. Wang, Luminosity Goals for a 100-TeV pp Collider, arXiv:submit/1238251 [hep-ph].

[18] D. Schulte, P. Lebrun et al., private communication (2015).

[19] C. Cook et al., Civil Engineering Optimisation Tool for the Study of CERN's Future Circular Collider, Proc. IPAC'15, Richmond (2015) 2079.

[20] B. Goddard, W. Bartmann, W. Herr, P. Lebrun, A. Milanese, Main changes to LHC layout for reuse as FCC-hh High Energy Booster, CERN-ACC-2015-030 (2015).

[21] A. Milanese, B. Goddard, M. Solfaroli Camillocci, Faster ramp of LHC for use as an FCC High Energy hadron Booster, CERN-ACC-2015-133 (2015).

[22] W. Bartmann et al., Transfer and Injection Process, Review of the FCC-hh Injection Energy, CERN, 16 October 2015, http: / / indico. cern. ch/event/449449.

[23] W. Chou, Circular accelerators: future machines and R\&D, Proc. EPS-HEP 2015, Vienna, July 2015.

[24] P. Lebrun, L. Tavian. Beyond the Large Hadron Collider: a first look at cryogenics for CERN future circular colliders, Proc. 25th International Cryogenic Conference \& International Cryogenic Materials Conference, Twente U., Enschede (2014).

[25] R. Kersevan, Arc vacuum design, absorbers \& shielding, FCC Week 2015, Washington, http://indico.cern.ch/event/340703/session/83/contribution/62 .

[26] R. Kersevan, Beam Screen Design and Cooling, Vacuum Aspects, Synchrotron Radiation, Review of the FCC-hh Injection Energy, CERN, 16 October 2015, http: //indico.cern.ch/event/449449. 
[27] O. Boine-Frankenheim, FCC-hh impedances and instabilities, Review of the FCC-hh Injection Energy, CERN, 16 October 2015, http: / / indico. cern. ch/event/449449.

[28] V. Shiltsev, Electron Lenses for Super-Colliders, Springer Verlag, Berlin, Heidelberg (2016).

[29] M. Bicer et al., First look at the physics case of TLEP, JHEP 01 (2014) 164.

[30] A. Blondel, Possibilities and conditions for very high precision electroweak measurements at TLEP3, 3rd EuCARD TLEP3 workshop, CERN, 10 January 2013, http://indico.cern.ch/event/222458.

[31] A. Bogomyagkov, E. Levichev, D. Shatilov. Beam-Beam Effects Investigation and Parameter Optimization for Circular e+e-Collider TLEP to study the Higgs Boson, Phys. Rev. ST Accel. Beams 17, 041004 (2014).

[32] M. Aiba, A. Saa Hernandez, F. Zimmermann, Top-up injection for FCC-ee, CERN-ACC-2015-065 (2015).

[33] M. Aiba, A. Saa Hernandez, Top-up Injection, Review of the FCC-ee Optics and Beam Dynamics, CERN, 14 October 2015, http: / / indico.cern. ch/event/448985.

[34] K. Oide, Optics Overview, Review of the FCC-ee Optics and Beam Dynamics, CERN, 14 October 2015 http: //indico.cern. ch/event/448985.

[35] A. Bogomyagkov, Crab Waist Interaction Region Designs and Solenoid Compensation, Review of the FCC-ee Optics and Beam Dynamics, CERN, 14 October 2015, http://indico.cern.ch/event/448985.

[36] K. Ohmi, F. Zimmermann, FCC-ee/CepC Beam-beam Simulations with Beamstrahlung, Proc. IPAC'14, Dresden (2014) 3766.

[37] D. Shatilov, Beam-Beam Effects in High-Energy Colliders: Crab Waist vs. Head-On, Proc. ICFA HF2014, Beijing (2014).

[38] A. Blondel, private suggestion following FCC Week 2015.

[39] J. Abelleira et al., A Large Hadron Electron Collider at CERN: Report on the Physics and Design Concepts for Machine and Detector, J. Phys. G 39 (2012) 075001.

[40] A. Butterworth, The RF System for FCC-ee, EPS-HEP 2015, Vienna, July 2015.

[41] D. Schoerling et al., Strategy for Superconducting Magnet Development for a Future Hadron-Hadron Circular Collider at CERN, Proc. EPS-HEP 2015, Vienna, July 2015.

[42] D. Tommasini, private communication (2015).

[43] A.V. Zlobin et al., Development and Test of a Single-Aperture 11 T Nb 3 Sn Demonstrator Dipole for LHC Upgrades, Published in IEEE Trans.Appl.Supercond. 23 (2013) 3, 4000904

[44] G.L. Sabbi et al., Performance Characteristics of Nb 3 Sn Block-Coil Dipoles for a 100 TeV Hadron Collider, Published in IEEE Trans.Appl.Supercond. 25 (2015) 3, 4001407.

[45] L. Brouwer, S. Caspi, S. Prestemon, Structural Analysis of an 18 T Hybrid CantedÚCosineÜTheta Superconducting Dipole, Published in IEEE Trans.Appl.Supercond. 25 (2015) 3, 4000404.

[46] L. Bottura, The RMC racetrack test magnet reaches a peak field of $16.2 T$, to be published in the CERN Courier (2015).

[47] I. Syratchev, Review of Efficient RF Sources, 2nd EuCARD2 Annual Meeting, Barcelona, 22 April 2015.

[48] Updated information is available on the FCC web site, http: / / cern. ch/fcc. 